\title{
Exploiting symmetries in polyhedral computations
}

\author{
Achill Schürmann
}

\begin{abstract}
In this note we give a short overview on symmetry exploiting techniques in three different branches of polyhedral computations: The representation conversion problem, integer linear programming and lattice point counting. We describe some of the future challenges and sketch some directions of potential developments.
\end{abstract}

\section{Introduction}

Symmetric polyhedra such as the Platonic and Archimedean solids have not only fascinated mathematicians since time immemorial. They occur frequently in diverse contexts of art and science. Less known to a general audience, but of great importance to modern mathematics and its applications, are higher dimensional analogues of these familiar objects. One standard description is as a set of solutions to a system of linear inequalities

$$
P=\left\{x \in \mathbb{R}^{n}: A x \leq b\right\},
$$

where $A$ is a real $m \times n$ matrix and $b \in \mathbb{R}^{m}$. A prominent example is the $n$-cube obtained by $2 n$ inequalities $\pm x_{i} \leq 1$. It has $2^{n}$ vertices (extreme points) with coordinates \pm 1 and its group of symmetries is the hyperoctahedral group of order $2^{n} n !$.

Linear models, and therefore polyhedra, are used in a wide range of mathematical problems and in applications such as transportation logistics, machine scheduling, time tabling, air traffic flow management and portfolio planning. They are central objects in Mathematical Optimization (Mathematical Programming) and are for instance heavily used in Combinatorial Optimization. Frequently studied symmetric polyhedra have names like "Travelling Salesman", "Assignment", "Matching" and "Cut". For these and further examples we refer to Sch03 and the numerous references therein. Over the years a rich combinatorial and geometric theory of polyhedra has been developed (see [Zie97, Grü03]). Symmetry itself is clearly a central topic in mathematics, and through the spread of computer algebra systems like GAP and MAGMA, sophisticated tools from Computational Group Theory are widely used today (see HEO05). Nevertheless, although many polyhedral problems are modeled with a high degree of symmetry, standard computational techniques for their solution do not take advantage of them. Even worse, often the used methods are known to work notoriously poorly on symmetric problems.

Achill Schürmann

Institute of Mathematics, University of Rostock, 18051 Rostock, Germany, e-mail: achill. schuermann@uni-rostock.de 
In this short survey we describe three main areas of polyhedral computations, in which the rich geometric structure of symmetric polyhedra can potentially be used for improved algorithms:

I: Polyhedral representation conversion using symmetry

II: Symmetric integer linear programming

III.Counting lattice points and exact volumes of symmetric polyhedra

There are multiple strong dependencies among the three topics and each one has its theoretical and algorithmic challenges as well as important applications. Before we take a closeup view on the three topics we give a brief introduction to the different types of polyhedral symmetries and how these can be determined and worked with.

\section{What are polyhedral symmetries?}

The symmetries of a polyhedron can be of a purely combinatorial nature or they can also have a geometric manifestation as affine symmetries, that is, as affine maps of $\mathbb{R}^{n}$ preserving the polyhedron. Among these symmetries are the "more visually accessible" isometries which are composed of translations, rotations and reflections. All of the symmetries of the $n$-cube for example are part of its isometry group. There exists a representation as a linear group in $\mathrm{GL}_{n}(\mathbb{R})$ and as a finite orthogonal group of isometries. However, if we perturb the defining inequalities a bit, all of these affine symmetries may be lost, while the new polyhedron is still combinatorially equivalent to a cube, sharing all of its combinatorial symmetries. These are defined as automorphisms of the polyhedral face lattice which encodes the combinatorial structure of a polyhedron. We refer to our survey $\mathrm{BDP}+12$ for further reading on these different types of polyhedral symmetry groups. The study of combinatorial lattices and their automorphisms is itself an active research area (see MS02]). The same is true for the study of possible isometry groups, respectively of finite orthogonal groups in $O_{n}(\mathbb{R})$. Their classification becomes in a way impractical for $n \geq 5$ (see [M037136]), despite the classification of finite simple groups (see $\left[\mathrm{CCN}^{+} 85\right]$ ). Even less is known about symmetry groups of polyhedra (see [Rob84]). Here, an "implication phenomenon" occurs, which has not much been studied so far. For instance, if a 4-gon has an element of order 4 among its affine symmetries, the 4-gon has to be the affine image of a square (2-cube), with an affine symmetry group of order 8 . These kind of implications clearly can potentially be exploited algorithmically, for example when detecting polyhedral symmetries.

It is important to note that the same abstract group can have different affine representations. We think that a key ingredient for future algorithmic improvements will be the use of geometric information coming with the affine representations of polyhedral symmetry groups. By a basic result in representation theory there is an invariant affine subspace $\mathcal{I}$ coming with each affine symmetry group. The polyhedron splits nicely into an invariant part $P \cap \mathcal{I}$ and symmetric slices orthogonal to it. These lie in fibers (pre-images) of the orthogonal projection onto $\mathcal{I}$. In a way, all of the symmetry is within these fibers.

Given a polyhedron with a group of symmetries, we say two vertices (or inequalities) are equivalent, if there exists a group element that maps one to the other. The set of vertices (and the set of facets / defining inequalities) splits into a number of orbits (disjoint sets of equivalent elements). For example, the $n$-cube has only one orbit of vertices and one orbit of facets. The same is true for all Platonic polyhedra and their higher dimensional analogues. In contrast, the Archimedean polyhedra 
like the soccer ball (truncated icosahedron) have more than one orbit of facets, but only one orbit of vertices. In all of these examples, their combinatorial symmetry group is equal to the group of affine symmetries. Its invariant affine subspace is a single point, the barycenter of the vertices.

In general, for a polyhedron $P$ with a group of affine symmetries, the vertices of the polyhedron split into orbits $O_{1}, \ldots, O_{l}$ and the invariant part $P \cap \mathcal{I}$ is equal to the convex hull conv $\left\{b_{1}, \ldots, b_{l}\right\}$, with $b_{i}=\left(\sum_{x \in O_{i}} x\right) /\left|O_{i}\right|$ being the barycenter of orbit $O_{i}$. This is due to two facts: The barycenter map, taking a point to the barycenter of its orbit, is an affine map. And second, the affine image of a convex hull of given points is equal to the convex hull of their affine images.

Thus working with the lower dimensional polyhedron $P \cap \mathcal{I}$ and its vertices gives us access to vertices of $P$. Orbits of integral points in $P$ have barycenters at specific locations in $P \cap \mathcal{I}$. For instance, if the group acts transitive on the coordinates of $\mathbb{R}^{n}$ then orbits have barycenters at integral multiples of $\left(\frac{1}{n}, \ldots, \frac{1}{n}\right)$. For more general coordinate permutations the barycenters form a scaled copy of a standard lattice (see [HRS12]).

\section{How to determine and work with symmetries?}

If the symmetries of the polyhedron are not known, the first difficulty is their determination and how to represent them. In general we like to work with as many symmetries as possible. However, the combinatorial symmetries can usually not be found without having full knowledge about the vertex-facet incidences of the polyhedron (see [KS03]). In contrast, the group of affine symmetries can be determined from the vertices or defining inequalities alone, by finding the automorphism group of an edge colored graph. If $P$ is given as the convex hull of its vertices $x_{1}, \ldots, x_{k}$, for instance, then the affine symmetry group can be obtained from the automorphism group of the complete graph with $k$ vertices and edge labels $x_{i}^{t} Q^{-1} x_{j}$, where

$Q=\sum_{i=1}^{k} x_{i} x_{i}^{t}$. For details and a proof we refer to BDS09. For further methods to compute polyhedral symmetry groups we refer to $\mathrm{BDP}+12$. Automorphism groups of graphs can be computed with software like bliss or [nauty. Given a polyhedral description, the affine symmetries can conveniently be obtained directly with our software [SymPol, which by now can also be used through polymake. For instance, given a polyhedron with its description contained in input-file, simply call:

sympol --automorphisms-only input-file

If the symmetry group of a polyhedron (or parts of it) are given as a permutation group, we can use sophisticated tools from Computational Group Theory. Each element of the group is then viewed as a permutation of the index set $\{1, \ldots, m\}$ of the input, for instance of $m$ defining inequalities. In practice, it is necessary to work with a small set of group generators if the group is large, and there are advanced heuristics to obtain such sets. Each face (and in particular each vertex) of a polyhedron is determined by a number of inequalities that are satisfied with equality; it can therefore be represented by a subset of $\{1, \ldots, m\}$. Given generators of a large permutation group and two subsets that represent faces, a typical computational bottleneck is to decide if both are in the same orbit. The fundamental data structures used for this in practice are bases and strong generating sets (BSGS, see Ser03, [HEO05]). Based on them, backtrack searches can be used to perform essential tasks, such as deciding on (non-)equivalence, obtaining stabilizers or fusing and splitting orbits. An elaborate version is the partition backtrack introduced by Leon [Leo91]. These backtracking methods work quite well in practice, although 
from a complexity point of view the mentioned problems are thought to be difficult (see Luk93]). Although computer algebra systems like [GAP and [MAGMA provide functions to work with permutation groups, for performance reasons it is often desirable to use problem specific code (see for example KÖ06). Nevertheless, all of these approaches, including [GAP] and [MAGMA, rely on efficient implementations of some partition backtrack. Therefore we have created a flexible $\mathrm{C}++$-implementation PermLib of Leon's partition backtrack (see Reh10a]) that can serve as a basis for the development of algorithms which combine tools from Computational Group Theory and Polyhedral Combinatorics. By now, PermLib has successfully been integrated into current versions of [SymPol, polymake and [SCIP].

\section{Representation Conversion}

By a fundamental theorem in polyhedral combinatorics, the Farkas-Minkowski-Weyl theorem, every polyhedron has a second representation as the convex hull of finitely many vertices (extreme points) and, in the unbounded case, some rays (see [Zie97]). Converting representations from inequalities to vertices (and rays), or vice versa, is a frequent task known as representation conversion problem (or convex hull problem). The importance of these conversions is due to the fact that some problems, like the maximization of a nonlinear convex function, are easy to solve in one presentation, but not in the other. Often, vertices represent objects that one would like to classify. These objects can be quite diverse, for instance perfect quadratic forms (see [DSV07]) or the elementary flux modes in biochemical reaction systems (see [SH94]). Representation conversions are also often used to analyze polyhedra in Combinatorial Optimization (see Sch03]). So far there exists no efficient algorithm for finding all the vertices of a polyhedron. In fact, the existence of such an algorithm appears to be unlikely, as it is NP-complete for polyhedra that are possibly unbounded (see $\left[\mathrm{KBB}^{+} 08\right.$ ). Nevertheless, several algorithms and implementations are widely used in practice (see for example [cdd] and [ $\mathrm{Irs}$ ] which are also available through polymake]).

Quite often one is only interested in one representative for each orbit of vertices (or inequalities) in a representation conversion. For example, when maximizing a nonlinear convex function on a polyhedron, or when vertices and inequalities in one orbit correspond to equivalent objects of some sort. Representation conversion up to symmetries has been considered in different contexts, and depending on the problem, different techniques have been successful. The most successful approaches currently known are the Incidence Decomposition Method and the Adjacency Decomposition Method (see for instance [CR96, [DFPS01, DFMV03, [DSV07, DSV09, [DSV10]). Both methods decompose the problem into a number of lower dimensional subproblems. They can be used recursively and can be parallelized (see [CR01, [DI07). Loosely speaking, the Incidence Decomposition Method fixes an orbit of the input, whereas the Adjacency Decomposition Method fixes an orbit of the output and then lists all "neighboring" orbits. For details we refer to our survey [BDS09]. We note that it is a priori not clear which method works best. We think best results can be achieved by a combination of different algorithms. All methods known so far do not use geometric insights and still rely on subproblem conversions that do not exploit available symmetry.

Our software [SymPol] and the experimental [GAP] package Polyhedral provide implementations of decomposition methods. These preliminary tools have already successfully been used in our own work (DSV07, [BBC 09], DSV09], [DSV10, DSE11), but also by others: For instance, Kumar Kum11 obtains a 
classification of elliptic fibrations that was previously impossible. Jacques Martinet writes in Mar03b] about the result in [DSV07]: "It seems plainly impossible to classify 8-dimensional perfect lattices." [SymPol can also be used to verify cumbersome calculations in proofs, like the edge-graph diameter analysis of the recently discovered, celebrated counterexamples to the Hirsch conjecture (see [San12, RS10]). For example, with the call

sympol --idm-adm-level 01 --adjacencies input-file

where input-file contains the 48 vertices of the 5-dimensional Santos prismatoid (see Table 1 in [San12]), SymPol returns a text file with a description of the adjacency graph of facets up to symmetry. Using a visualization tool like [Graphviz, the produced textfile, say adjacencies.dot, can then easily be turned into an image with a command like

neato -Tpng -o adjacencies.png adjacencies.dot

From the obtained image (see figure) it is easily verified that the shortest path from facet 1 to facet 12 is of length 6 , which is the key calculation in the proof of [San12].

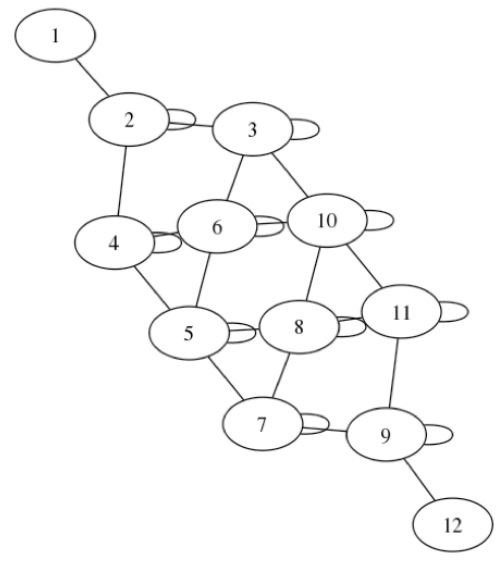

Let us make a remark on the increasing importance of mathematical software in general: As sophisticated computational tools become an increasingly important basis for high-level mathematical research, their creation also becomes an increasingly important service to the mathematical community. More and more mathematicians use computers in the creative process and to verify standard parts of difficult larger proofs (see e.g. Hal05, CK09, Hal11]). Timothy Gowers Gow00 even guesses that at the end of the 21st century, computers will be better than humans in proving theorems. Although we would not go as far as Gowers, we are convinced that in the future, parts of proofs will routinely be performed by computers. With a symbiosis of human and computer reasoning we will see substantial advances in mathematical problems. In this way reliable mathematical software becomes an increasingly important part of mathematics itself.

\section{Challenges}

One of the most challenging polyhedral conversion problems arises in conjunction with lattice sphere packing problem, a classical problem in the Geometry of Numbers. Since its solution up to dimension 8 almost 80 years ago, it is still open in dimension $n \geq 9$, with the exception of dimension 24 (see [Mar03b, CK09]). One way to approach this problem is via finding the vertices of a locally polyhedral 
object known as Ryshkov polyhedron (see [Sch09a for details). The currently open 9-dimensional case leads to a challenging representation conversion problem of a 45dimensional Ryshkov polyhedron. Main difficulties here come from faces that carry the symmetries of the exceptional Weyl group $E_{8}$. We think that this problem is a particular nice test case, as all finite rational matrix groups appear as stabilizers of faces in the Ryshkov polyhedron. So in a way, this challenging representation conversion problem gives a universal test case for any future algorithmic advances.

\section{Integer Linear Programming}

Linear programming is the task of maximizing (or minimizing) a linear function on a polyhedron given by linear inequalities. It serves as a fundamental basis for theory and computations in Mathematical Optimization (see Tod02). In Integer linear programming vectors to be optimized are restricted to integers (number of goods, etc.) or even to 0/1 entries (encoding a simple yes-no-decision). Integer linear programming is widely used in practical applications. In fact, "the vast majority of applications found in operations research and industrial engineering involve the use of discrete variables in problem formulation" (from a book review of Wol98). In many of these problems the involved polyhedra have symmetries (see [Sch03]). From a complexity point of view, integer programming is NP-complete (see Kar72), whereas linear programming can be solved in polynomial time. For fixed dimension, polynomial time algorithms are known for integer linear programming (see [Len83]).

A linear programming problem max $c^{t} x$ with $x \in P$ is invariant with respect to a linear symmetry group $\Gamma \leq \mathrm{GL}_{n}(\mathbb{R})$, if the polyhedron $P$ and the utility vector $c \in \mathbb{R}^{n}$ are preserved by it, that is, if $\Gamma P=P$ and $\Gamma c=c$. Any solution to the linear program, its orbit and the barycenter of its orbit lie in the same hyperplane orthogonal to the utility vector and therefore have the same utility value. Due to the convexity of polyhedra, the barycenter is also a feasible solution. As it lies in the invariant linear subspace $\mathcal{I}$ of $\Gamma$, the linear programming problem always has a solution attained within $\mathcal{I}$. Thus it is possible to solve the lower-dimensional linear program max $c^{t} x$ with $x \in P \cap \mathcal{I}$. Such symmetry reductions are often referred to as "dimension reduction" or "variable reduction". The symmetries of an integer linear program are more restrictive, as also $\mathbb{Z}^{n}$ has to be left invariant by the group $\Gamma$.

Exploiting symmetries in integer programming is much more difficult than in linear programming. In fact, symmetries are rather problematic, as standard methods like branch-and-bound or branch-and-cut (see [Sch86]) have to solve many equivalent sub-problems in such cases. In contrast to linear programming, it is not possible to simply consider the intersection with the invariant affine subspace, as integral solutions can lie outside. Nevertheless, in recent years it has been shown that it is possible to exploit symmetries in integer programming; see for example [Mar03a], [Fri07, BM08, KP08, OLRS11, LMT09. These specific methods fall into two main classes: They either modify the standard branching approach, using isomorphism tests or isomorphism free generation to avoid solving equivalent subproblems; or they use techniques to cut down the original symmetric problem to a less symmetric one, which contains at least one element of each orbit of solutions. For further reading we refer to the excellent survey Mar10.

As many real world applications can be modeled as (mixed) integer programming problems, a variety of professional software packages are available. Two of the leading ones, CPLEX and Gurobi, have by now included some techniques to avoid or use symmetry. Unfortunately it is publicly not known what exactly is done. 
None of the known methods uses the rich geometric properties of the involved symmetric polyhedra. Using the fact that solutions are "near" the linear invariant subspace, it is possible to do better. For the special case of a one dimensional invariant subspace, with the full symmetric group $S_{n}$ acting transitively on the coordinates in $\mathbb{R}^{n}$, this is shown in BHJ11. We have highly promising results with a generalization to arbitrary symmetries in [HRS12]: In particular for direct products of symmetric groups, we not only beat state-of-the-art professional solvers, but even solve a challenging, previously unsolved benchmark problem from [MIPLIB] (instance toll-like).

The main ingredient is the observation that any feasible integer linear programming problem with a non-trivial affine symmetry group contains certain core-sets of integral vectors that can be used as a kind of test-set, that is, if non of the points from the core-set is contained in the feasible region, no integral point is. Assume $\Gamma \leq \mathrm{GL}_{n}(\mathbb{R})$ is a linear representation of a given symmetry group and $\mathcal{I}$ denotes its invariant linear subspace containing the utility vector $c$. Then we say an integral point $z$ in a fiber (pre-image) of the orthogonal projection onto $\mathcal{I}$ is in the core-set of the fiber if the convex hull of its orbit $\Gamma z$ does not contain any integral points aside those of the orbit itself. Then, by definition, representatives of each orbit $\Gamma z$ in the core-set can be used as a test-set for feasibility of a fiber.

Using the Flatness Theorem (from the Geometry of Numbers), it can be shown that core-sets are finite for irreducible groups. For direct products of symmetric groups acting on some of the coordinates, the test-set containing only representatives of orbits even reduces to a single point. Besides that not much is known so far about core-sets. Nevertheless, we think that they will serve as a powerful tool in the design of new algorithms for symmetric integer linear programs.

\section{Challenges}

Challenging examples of symmetric integer linear programs can be found in benchmark libraries like [MIPLIB]. These problems come from diverse contexts and have not been chosen to be particular symmetric. Nevertheless many symmetries can be found and exploiting them algorithmically, beating state-of-the-art commercial solvers, remains a challenging test case for future advances.

Some particular symmetric integer linear programming problems coming from difficult combinatorial problems in mathematics have been collected (and worked on) by Francois Margot at symlp (see for instance also Mar03a and [BM08]). As these problems have been intensively worked on, improving on the currently known results is certainly a hard problem. So this gives a very good benchmark for future improvements as well.

\section{Lattice Point Counting and Exact Volumes}

Often it is desirable to know how many integral solutions there are to a system of linear inequalities. Such problems occur frequently in Combinatorics (see [Sta97]) but also in disciplines such as representation theory (Kostka and Littlewood-Richardson coefficients, see [BZ01] and [KT99]), in statistics (contingency tables, see [DS98]), in voting theory (see WP07 and GL11), and even in compiler optimization (see Graphite). We refer to DeL05 for an overview. Counting lattice points is moreover intimately related to integer linear programming (see [Las09]). 
By a breakthrough result of Barvinok [Bar94] in the 1990s, counting lattice points inside a rational polyhedron can be done in polynomial time for fixed dimension. His ideas are based on evaluating "short rational generating functions" and on constructing unimodular triangulations. His algorithm has been implemented in LattE and barvinok. The same applies to a slightly more general setting, in which one considers a one-parameter family of dilations $\lambda P$, with $P$ a rational polyhedron and $\lambda$ an integer. By a theory initiated by Ehrhart in the 1960s (see [Ehr67], [BR07]), it is possible to obtain the number of integral points in the dilate $\lambda P$ by a quasi-polynomial in $\lambda$, with its degree equal to the dimension of $P$. A quasi-polynomial $p$ is determined by a finite number of polynomials $p_{i}, i=0, \ldots, k$, via the setting $p(\lambda)=p_{i}(\lambda)$ for all $\lambda$ congruent to $i \bmod k$. In case of $P$ being integral, the Ehrhart quasi-polynomial simply is a polynomial in $\lambda$. In general, the quasi-polynomial can also be computed in polynomial time by Barvinok methods (see Bar08]). Often, the main interest is only in the leading coefficient of the Ehrhart quasi-polynomial, which is the volume of $P$. Computing the volume itself is already a \#P-hard problem (see [DF88, BW91]).

Despite the fact that many counting problems have plenty of symmetries, they have not been exploited systematically so far. In other words, exploiting symmetry in lattice point counting, or more generally in Ehrhart theory, is a vastly open subject. For volume computations the situation seems a bit better. For very special volume computations symmetry can be exploited (see DSV09]). However, there is still a huge potential for improved methods. Many of the difficulties originate from the fact that the "Barvinok methods" used to solve them rely on unimodular triangulations of polyhedral cones that usually do not inherit the symmetry of the polyhedron. New roads will have to be taken here.

In $[$ Sch12 it is shown that it is possible to exploit symmetries by using a decomposition into symmetric slices, together with a weighted Ehrhart theory. The theoretical background and first implementations for such a theory have just recently been developed (see $\left.\mathrm{BBD}^{+} 11 \mathrm{~b}, \mathrm{BS12}\right)$. The polyhedral decomposition used in $\mathrm{Sch} 12$ is rather special: There is a linear invariant part and symmetric slices orthogonal to it, which are cross-products of regular simplices (simplotopes). A generalization to other decompositions is easily obtained, whenever there is a decomposition into an invariant part and slices orthogonal to it for which the Ehrhart quasi-polynomial is known. Note that the decomposition can easily be obtained in a automated way, as the invariant part is the intersection of the given polyhedron with the affine space fixed by its symmetry group.

For exploiting symmetry in corresponding volume computations, the integration of polynomials over a polyhedron is used. Using Brion-Lawrence-Varchenko theory, this can efficiently be done by integrating sums of powers of linear forms (see $\left.\mathrm{BBD}^{+} 11 \mathrm{a}\right]$. The new decomposition approach of [Sch12] also allows to obtain exact volumes that have not been computable before. This is demonstrated on three well studied examples from Social Choice theory, which give the exact likelihood of certain election outcomes with four candidates that were previously known for three candidate elections only (see [GL11]).

\section{Challenges}

In Social Choice theory we face a large amount of challenging problems related to probability calculations of voting situations with four and more candidates. The only known results in the context of the "polyhedral model" (IAC hypothesis) appear 
to be those in [Sch12, which are obtained by exploiting polyhedral symmetry as described above.

A challenging benchmark volume computation that several researchers previously have looked at is the volume of the Birkhoff polytope $B_{n}$ (also known as perfect matching polytope of the complete bipartite graph $K_{n, n}$ ). The current known record is the volume of $B_{10}$ due to Beck and Pixton [BP03, using a complex-analytic way to compute the Ehrhart polynomial. The computation of the volume of $B_{11}$ would certainly be quite a computational achievement.

\section{Conclusions}

We expect that symmetry exploiting techniques for polyhedral computations can be vastly improved by using geometric properties that come with affine symmetries of polyhedra. Concentrating on improvements in polyhedral computations with affine symmetries is practically no restriction: If a polyhedron is given, either by linear inequalities or vertices and rays, the affine symmetries of the (potentially larger) combinatorial symmetry group are practically the only ones we can compute.

For polyhedral representation conversions we see potential in enhancing decomposition methods through the use of geometric information like fundamental domains, classical invariant theory and symmetric polyhedral decompositions. For integer linear programming we expect that a new class of algorithms based on the concept of core points will help to exploit symmetry on difficult symmetric integer linear programming problems. For exact volume computations and counting of lattice points, there is still a lot of potential for new ideas using symmetry. Overall, we think symmetry should be exploitable whenever it is available. For this goal to be reached there seem still quite some efforts necessary though.

\section{References}

$\mathrm{BBD}^{+}$11a. V. Baldoni, N. Berline, J.A. De Loera, M. Köppe and M. Vergne, How to integrate a polynomial over a simplex, Math. Comp. 80 (2011), no. 273, 297-325.

$\mathrm{BBD}^{+} 11 \mathrm{~b} . \quad$ Computation of the highest coefficients of weighted Ehrhart quasi-polynomials of rational polyhedra, Found. Comput. Math., 2011, to appear, preprint at arxiv:1011.1602

$\mathrm{BBC}^{+}$09. B. Ballinger, G. Blekherman, H. Cohn, N. Giansiracusa, E. Kelly and A. Schürmann, Experimental study of energy-minimizing point configurations on spheres, Experiment. Math. 18 (2009), 257-283.

Bar94. A. Barvinok, A polynomial time algorithm for counting integral points in polyhedra when the dimension is fixed, Math. Oper. Res. 19 (1994), 769-779.

Bar08. Integer points in polyhedra, European Mathematical Society (EMS), 2008.

BP03. M. Beck and D. Pixton, The Ehrhart polynomial of the Birkhoff polytope, Discrete Comput. Geom., 30 (2003), 623-637; see also the update in arxiv:math/0305332

BR07. M. Beck and S. Robins, Computing the continuous discretely, Springer, 2007.

BZ01. A.D. Berenstein and A.V. Zelevinsky, Tensor product multiplicities, canonical bases and totally positive varieties, Invent. Math. 143 (2001), 77-128.

BHJ11. R. Bödi, K. Herr and M. Joswig, Algorithms for highly symmetric linear and integer programs, Math. Program., Ser. A, 2012, to appear, published online, http://dx.doi. org/10.1007/s10107-011-0487-6

BDP +12 . D. Bremner, M. Dutour Sikirić, D. V. Pasechnik, T. Rehn, A. Schürmann, Computing symmetry groups of polyhedra, preprint at arxiv:1210.0206

BDS09. D. Bremner, M. Dutour Sikirić and A. Schürmann, Polyhedral representation conversion up to symmetries, in CRM Proceedings \& Lecture Notes 48, AMS, 2009, pp. $45-71$.

BW91. G. Brightwell and P. Winkler, Counting linear extensions, Order 8 (1991), 225-242. 
BS12. W. Bruns and C. Söger The computation of generalized Ehrhart series in Normaliz, preprint at arxiv:1211.5178.

BM08. D.A. Bulutoglu and F. Margot, Classification of orthogonal arrays by integer programming, Journal of Statistical Planning and Inference 138 (2008), 654-666.

CR96. T. Christof and G. Reinelt, Combinatorial optimization and small polytopes, Top 4 (1996), 1-64.

CR01. Decomposition and parallelization techniques for enumerating the facets of combinatorial polytopes, Internat. J. Comput. Geom. Appl. 11 (2001), 423-437.

CK09. H. Cohn and A. Kumar, Optimality and uniqueness of the Leech lattice among lattices, Ann. of Math. 170 (2009), 1003-1050.

$\mathrm{CCN}^{+} 85$. J.H. Conway, R.T. Curtis, S.P. Norton, R.A. Parker and R.A. Wilson, Atlas of finite groups, Oxford University Press, 1985.

DeL05. J.A. De Loera, The many aspects of counting lattice points in polytopes, Math. Semesterber. 52 (2005), 175-195.

DFMV03. A. Deza, K. Fukuda, T. Mizutani and C. Vo, On the face lattice of the metric polytope, in Discrete and computational geometry, LNSC 2866, Springer, 2003, pp. 118-128.

DFPS01. A. Deza, K. Fukuda, D. Pasechnik and M. Sato, On the skeleton of the metric polytope, in Discrete and computational geometry, LNSC 2098, Springer, 2001, pp. 125-136.

DI07. A. Deza and G. Indik, A counterexample to the dominating set conjecture, Optimization Letters 1-2 (2007), 163-169.

DS98. P. Diaconis and B. Sturmfels, Algebraic algorithms for sampling from conditional distributions, Ann. Statist. 26 (1998), 363-397.

DSE11. M. Dutour Sikirić, A. Schürmann and G.Ellis, On the integral homology of $P S L_{4}(\mathbb{Z})$ and other arithmetic groups, J. Number Theory 131 (2011), 2368-2375.

DSV07. M. Dutour Sikirić, A. Schürmann and F. Vallentin, Classification of eight dimensional perfect forms, Electron. Res. Announc. Amer. Math. Soc. 13 (2007), 21-32.

DSV09. M. Dutour Sikirić, A. Schürmann and F. Vallentin, Complexity and algorithms for computing Voronoi cells of lattices, Math. Comp. 78 (2009), 1713-1731.

DSV10. M. Dutour Sikirić, A. Schürmann and F. Vallentin, The contact polytope of the Leech lattice, Discrete Comput. Geom. 44 (2010), 904-911.

DF88. M.E. Dyer and A.M. Frieze, On the complexity of computing the volume of a polyhedron, SIAM J. Comput. 17 (1988), 967-974.

Ehr67. E. Ehrhart, Sur un problème de géométrie diophantienne linéaire. I. Polyèdres et réseaux, J. Reine Angew. Math. 226 (1967), 1-29.

Fri07. E.J. Friedman, Fundamental domains for integer programs with symmetries, in Combinatorial optimization and applications, LNSC 4616, Springer, 2007, pp. 146-153.

GL11. W.V. Gehrlein and D. Lepelley, Voting paradoxes and group coherence, Springer, 2011.

Gow00. W.T. Gowers, Rough structure and classification, in Visions in mathematics - Towards 2000, Birkhäuser, 2000, pp. 79-117.

Grü03. B. Grünbaum, Convex Polytopes, 2nd ed. (V. Kaibel, V. Klee and G. M. Ziegler, eds.), Springer, 2003.

Hal05. T.C. Hales, A proof of the Kepler conjecture, Ann. of Math. 162 (2005), 1065-1185.

Hal11. _ The Strong Dodecahedral Conjecture and Fejes Toth's Conjecture on Sphere Packings with Kissing Number Twelve, preprint at arxiv:1110.0402

HRS12. K. Herr, T. Rehn and A. Schürmann, Exploiting Symmetry in Integer Convex Optimization using Core Points, preprint at arxiv:1202.0435

HEO05. D.F. Holt, B. Eick and E.A. O'Brien, Handbook of computational group theory, Chapman \& Hall/CRC, 2005.

KP08. V. Kaibel and M.E. Pfetsch, Packing and partitioning orbitopes, Math. Program., Ser.A 114 (2008), 1-36.

KS03. V. Kaibel and A. Schwartz, On the complexity of polytope isomorphism problems, Graphs Combin. 19 (2003), 215-230.

Kar72. R.M. Karp, Reducibility among combinatorial problems, in Complexity of Computer Computations, Plenum, 1972, pp. 85-103.

KÖ06. P. Kaski and P.R.J. Östergård, Classification algorithms for codes and designs, Springer, 2006.

$\mathrm{KBB}^{+} 08$. L. Khachiyan, E. Boros, K. Borys, K. Elbassioni and V. Gurvich, Generating all vertices of a polyhedron is hard, Discrete Comput. Geom. 39 (2008), 174-190.

KT99. A. Knutson and T. Tao, The honeycomb model of $\mathrm{GL}_{n}(\mathbb{C})$ tensor products I. Proof of the saturation conjecture, J. Amer. Math. Soc. 12 (1999), 1055-1090.

Kum11. A. Kumar, Elliptic fibrations on a generic Jacobian Kummer surface, J. Algebraic. Geom., 2012, to appear, preprint at arxiv:1105.1715

Las09. J.B. Lasserre, Linear and Integer Programming vs Linear Integration and Counting, Springer, 2009. 
Len83. H.W. Lenstra Jr., Integer programming with a fixed number of variables, Math. Oper. Res. 8 (1983), 538-548.

Leo91. J.S. Leon, Permutation group algorithms based on partitions. I. Theory and algorithms, J. Symbolic Comput. 12 (1991), 533-583.

LMT09. J. Linderoth, F. Margot and G. Thain, Improving bounds on the football pool problem via symmetry reduction and high-throughput computing, INFORMS Journal on Computing 21 (2009), 445-457.

Luk93. E.M. Luks, Permutation groups and polynomial-time computation, in Groups and computation, AMS, 1993, pp. 139-175.

Mar03a. F. Margot, Exploiting orbits in symmetric ILP, Math. Program., Ser.B 98 (2003), $3-21$.

Mar10. _ Symmetry in integer linear programming, in 50 years of integer programming, Springer, 2010, pp. 647-686.

Mar03b. J. Martinet, Perfect lattices in Euclidean spaces, Springer, 2003.

MS02. P. McMullen and E. Schulte, Abstract regular polytopes, Cambridge University Press, 2002.

OLRS11. J. Ostrowski, J. Linderoth, F. Rossi and S. Smriglio, Orbital branching, Math. Program., Ser.A 126 (2011), 147-178.

Reh10a. T. Rehn, Fundamental Permutation Group Algorithms for Symmetry Computation, Diploma Thesis in Computer Science, OvG University Magdeburg, 2010.

RS10. T. Rehn and A. Schürmann, $C++$ tools for exploiting polyhedral symmetries, in Mathematical Software - ICMS 2010, LNCS 6327, Springer, 2010, pp. 295-298.

Rob84. S.A. Robertson, Polytopes and symmetry, Cambridge University Press, 1984.

San12. F. Santos, A counterexample to the Hirsch conjecture, Ann. of Math., 176 (2012), 383-412.

Sch86. A. Schrijver, Theory of linear and integer programming, Wiley, 1986.

Sch03. _ Combinatorial optimization. Polyhedra and efficiency, Vol. A, B, C, Springer, 2003.

Sch09a. A. Schürmann, Computational geometry of positive definite quadratic forms, AMS, 2009.

Sch12. _ Exploiting Polyhedral Symmetries in Social Choice, Social Choice and Welfare, 2012, to appear, published online, http://dx.doi.org/10.1007/s00355-012-0667-1 preprint at arxiv:1109.1545.

SH94. S. Schuster and C. Hlgetag, On elementary flux modes in biochemical reaction systems at steady state, Journal of Biological Systems 2 (1994), 165-182.

Ser03. Á. Seress, Permutation group algorithms, Cambridge University Press, 2003.

Sta97. R.P. Stanley, Enumerative combinatorics, vol. 1, Cambridge University Press, 1997.

Tod02. M.J. Todd, The many facets of linear programming, Math. Program., Ser. B 91 (2002), $417-436$.

WP07. M.C. Wilson and G. Pritchard, Probability calculations under the IAC hypothesis, Math. Social Sci. 54 (2007), 244-256.

Wol98. L.A. Wolsey, Integer programming, John Wiley \& Sons Inc., 1998.

Zie97. G.M. Ziegler, Lectures on polytopes, Springer, 1997.

\section{SOFTWARE AND WeBPAGES}

$\begin{array}{ll}\text { barvinok. } & \text { Counting lattice points, http://www.kotnet.org/ skimo/barvinok/ } \\ \text { bliss. } & \text { Graph and labeling automorphisms, http://www.tcs.hut.fi/Software/bliss/ } \\ \text { cdd. } & \text { Double Description, http://ww.ifor.math.ethz.ch/ fukuda/cdd_home/ } \\ \text { CPLEX. } & \text { Mathematical programming technology, http://www.ilog.com/products/cplex/ } \\ \text { GAP. } & \text { Groups, Algorithms, Programming, http://ww.gap-system.org/ } \\ \text { Graphite. } & \text { High-level memory optimizations, http://gcc.gnu.org/wiki/Graphite } \\ \text { Graphviz. } & \text { Graph Visualization Software, http://www.graphviz.org/ } \\ \text { Gurobi. } & \text { High-end libraries for math programming, http://www.gurobi.com/ } \\ \text { LattE. } & \text { Lattice point count, volumes and integrals, http://www.math.ucdavis.edu/latte/ } \\ \text { lrs. } & \text { Lexicographic reverse search, http://cgm.cs.mcgill.ca/ avis/C/lrs.html } \\ \text { MAGMA. } & \text { Computational Algebra System, http://magma.maths.usyd.edu.au/magma/ } \\ \text { MO37136. } & \text { Classification of finite groups of isometries,http://mathoverflow.net/questions/ } \\ & \text { 37136/classification-of-finite-groups-of-isometries } \\ \text { MIPLIB. } & \text { Mixed Integer Problem Library, http://miplib.zib.de/ }\end{array}$


nauty. Graph and labeling automorphisms, http://cs.anu.edu.au/ bdm/nauty/

PermLib. Permutation groups library, http://www.geometrie.uni-rostock.de/software/

Polyhedral. A polyhedral GAP package, http://www.liga.ens.fr/ dutour/Polyhedral/

polymake. A framework for analyzing convex polytopes, http://polymake.org/

SCIP. Solving Constraint Integer Programs, http://scip.zib.de/

symlp. Symmetric IP problems, http://wpweb2.tepper.cmu.edu/fmargot/lpsym.html

SymPol. Symmetric polyhedra toolkit, http://www.geometrie.uni-rostock.de/software/ 